\title{
Transfer of Large Equine Embryos in Arabian Mares
}

\author{
M.K. Derbala ${ }^{1 *}$, M.S.S. Abdou ${ }^{2}$ \\ ${ }^{1}$ Animal Reproduction Research Institute-ARC-Egypt \\ ${ }^{2}$ Theriogenology Dept. Faculty of Veterinary Medicine, Cairo University, Egypt
}

*Corresponding Author: M.K. Derbala, Animal Reproduction Research Institute-ARC-Egypt, Email: mohamed_equine@yahoo.com

\begin{abstract}
In the vast majority of equine embryo transfer programs, flushing takes place on days 6, 7 or 8 post ovulation. In the present study, embryos could, instead, be obtained on days 10-11 after ovulation. For this purpose, 36 Arabian mares (7-24 years old) were used as donors for embryos and 6 mares were kept as control. Of the 36 donor animals, 2 mares died suddenly and flushing was carried out after excision of the uterus.

Recipient mares $(N=70)$ aged $5-10$ years, and were kept in embryo transfer facility. The degree of synchronization was -4 to -6 days.

The procedure used depended on flushing of the donor mares after detection of embryonic sac using ultrasonography. Large pore AI catheters and external sheath of double guarded uterine swabs were used in the process of embryo transfer. A controllable manual pipette was used in the control process of loading, washing and transfer. This method overcame the problem of burst of large embryos.

A high recovery $(94.4 \%)$ and pregnancy $(73.5 \%)$ rates could be obtained. Results have also shown that higher pregnancy rate was obtained with recipient mares on day 4 post ovulation, whereas lower pregnancy rates was found in recipient mares on day 6 post ovulation.

In conclusion, this study demonstrated that there was a possibility of embryo transfer on day 10-11 post ovulation i.e. after embryo detection with ultrasound scanning. This method permits flushing of mare's uterus after death on 10-11 days of pregnancy for maximum exploitation of the donor mare. Furthermore, concerning mares with a history of low embryo recovery flushing did not take place until the embryo was detected with ultrasound so as to save flushing media and number of flushes.
\end{abstract}

Keywords: Embryo transfer; Equine; Large embryos; Mare; Synchrony

\section{INTRODUCTION}

The use of embryo transfer and development of embryo technology for the horse have increased steadily over the past two decades (Squires, Carnevale, McCue and Bruemmer; 2003). Embryo transfer (ET) is now generally accepted as a valuable tool for increasing the number of progeny from genetically valuable mares, for producing foals from competing mares without interrupting their sporting careers, and for obtaining foals from mares incapable of carrying a pregnancy to term(Stout; 2006).

Additionally, ET can be performed on young mares as a method to get them into production 1 or 2 years earlier than conventional method (Camillo, Vannozi, and Rota 2002).

Embryos are usually recovered on day 6,7 or 8 (considering day 0 is the day of ovulation) from naturally single or occasionally multiple ovulations. Embryos are recovered 1 day later if the mare has been inseminated using frozen semen rather than fresh or cooled semen (Mckinnon and Squires, 2007). Recovery rates on days 7,8 and 9 post ovulation are quite similar, but recovery of 6 day embryos is slightly lower which explained by inability to identify the embryo in recovery medium, loss of embryo during recovery procedure due to its small size, inability to obtain the embryo in the uterine flush because of its greater specific gravity or failure of some embryos to descend into the uterus by day 6 (Iuliano, Squires and Cook, 1985).

The major factor affecting embryo recovery is the mare's reproductive history. Older mares with poor reproductive histories produce fewer embryos. Causes of reduced embryo recovery from these older mares include uterine and oviductal pathology and increases early embryonic death (Ball, Little, Weber, and Wood, 1989). 
Limited studies are available addressing the effect of embryo age on pregnancy rate (Allen, Stewart, Trounson, 1976; Steffenhagen, Pineda and Ginther, 1972; Iuliano, 1983; Iuliano, Squires and Cook, 1985). However, in recent years there has been a tendency by some veterinarians to flush mares on day 8 or 9 so that the embryo may be visualized in the filter, enabling the flush to be stopped after just one flush rather than three that are recommended. This practice can easily leave a twin embryo in the uterus (David Hartman, 2011). However, the team in GoulburnValley Equine Hospital $(\mathrm{GVEH})$ produced a foal that transferred at 10 days old. Once they identify the mare pregnant using ultrasonography, they then harvest and transfer the embryo. The catheter used to flush the mare is necessary large(Mckinnon and Squires, 2007).Additionally, Vogelsang et al.(1985) reported no pregnancies after transfer of 9 and 10 days old embryos, whereas pregnancy rates of $61 \%, 55 \%$, and $25 \%$ were observed after transfer of days 5, 7, and 8 embryos, respectively. Varied results were found by Fleury et al (1989) who achieved 69\% pregnancy rate after transfer of 16 embryos of day 9 old. It is noteworthy that these two studies were performed while the procedure of equine embryo transfer was still primitive and had not been in practice for a long time, which could have contributed to the disparate results.

Jacob et al (2012) have recently shown that embryo recovery rates between Days 7 and 10 were similar and acceptable (e.g., 63\%), the degree of synchronization between donor and recipient mares does not need to be as restricted as previously reported in horses. There was acceptable pregnancy rates (e.g., 70\%) obtained even when recipient mares ovulated 4 to 5 days after the donors. Similar pregnancy rates were obtained when embryos transferred into recipient mares with wide range of ovulation (days 3 to 8 post ovulation). Additionally, transfer of day 7 embryos produced higher pregnancy rates when compared with Days 8 and 9 embryos.

There are few studies concerning transfer of 10 days embryo. Wilsher et al (2010) reported that transfer of day 10 embryos to asynchronous recipient mares could be beneficial in the management of conventional equine embryo transfer programs and may provide a useful model for verifying of maternal effects on early embryogenesis. Although they mentioned that the problematic aspect of the transfer of large (day 10) embryos is the use of transfer catheters provided with an internal diameter $\geq 5 \mathrm{~mm}$; the capillary forces required to hold the embryo in its transfer medium in set columns within the catheter do not achieved in these larger bore catheters. Furthermore, Wilsher et al (2010) couldn't recover embryos $>5 \mathrm{~mm}$ despite of repeated time numbers of flushing.

Squires et al.(2003) indicated that the vast majority of embryos are transferred using nonsurgical method into synchronized recipients. For nonsurgical transfer, embryos are loaded into $0.25-0.5 \mathrm{ml}$ straws and inserted into the body of the uterus using an embryo transfer gun. Alternatively, the embryo is loaded into a flexible insemination pipette that is covered with a protective sheath and introduced through the cervix into the body of the uterus.

\section{Materials ANd Methods}

\subsection{Donor Mares}

This study was carried out during September, 2015-October 2016. The donor mares $(\mathrm{N}=36)$ were straight Egyptian mares weighing between $350-400 \mathrm{~kg}$ and between 7-24 years old. In addition to 6 mares were kept as a control. The mares were fed green fodders mixed with hay, and were given barely, access to mineral salt and fresh water was provided ad libitum.

\subsection{Ultrasonographic Scan}

Transrectal ultrasonography was applied for donor mares for determination of proper time of breeding as well as for detection of ovulation time (using 7.5 Mhz linear endorectal probe, MYLAB 30, Italy and Sonoscape; China)

\subsection{Insemination and Breeding Management}

Natural or artificial breeding has been done. Stallions used were kept in the same ET facility. These stallions were examined periodically for assessment of semen quality. For increasing embryo recovery rates, mares were treated for infertility problems (using of ecbolics, uterine lavage, intrauterine antibiotics and corticosteroids).

\subsection{Recipient Mares}

Recipient mares $(\mathrm{N}=70)$ were kept in the ET facility. They were between 5 and 10 years old 
and weighed around $350-450 \mathrm{~kg}$. All mares were fed green fodder, hay and concentrates. Estrous cycles were monitored using transrectal palpation and ultrasonography to synchronize ovulation between the donor and recipient mares. The degree of synchronization was -4 to - 6 days (recipient ovulated 4-6 days after donor). The degree of synchrony was achieved using Cloprostenol (Estrumate; MSD, Animal Health) as a luteolytic agent, and 1500 IU of hCG (Epifasi; EPICO, Egypt) as an ovulatory inducing agent.

\subsection{Collection and Evaluation of Embryos}

Beginning from day 9 post ovulation, the uterus of donor mares was scanned daily using $7.5 \mathrm{Mhz}$ transrectal ultrasound probe (MYLAB 30, Italy and Sonoscape A5v, China). The uterine flushes were performed with detection of embryonic sac

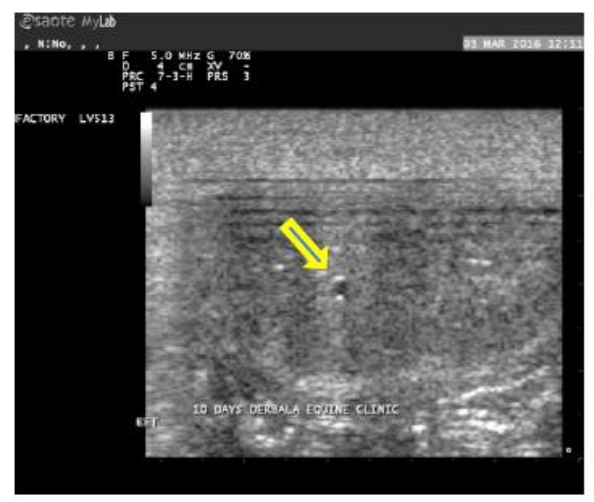

Fig (1-A). Detection of embryo with ultrasound scanning in a donor mare for ET on day 10 post ovulation

\subsection{Transfer of Embryos}

After detection of the embryo in the EmSafe filter, it was picked up and washed in a holding media. The embryo was loaded in a catheter using a special kind of pipette filler (A manual propipetter adjusted by turning the wheel with the thumb) to control suction and release to avoid rupture of the embryo (Fig. 2-a). A.I catheters, modified A.I, ET catheters and external protecting sheath of double guarded uterine swab have been used, respectively, for embryo transfer according to embryo size (Fig. 2-b). Contamination was minimized by enclosing the AI catheter in a sterile sheath. Passage of the pipette through the cervix was mainly performed transvaginally. The embryo was deposited into the uterus and the catheter and sheath were withdrawn from the mare. by ultrasound scan (Fig. 1-a, 1-b). After washing the perineal area with mild soap and povidone iodine solution, a 36 Foley catheter (Minitüb COP, Germany) was finger- guided through the cervix into the uterine body. Once in, the cuff on the end of the catheter was inflated with $30 \mathrm{ml}$ of Ringer's solution then pulled caudally to ensure a tight seal against the internal os of the cervix. Lactated Ringer's solution $(1 \mathrm{~L})$ was infused through the catheter and into the uterus then drained into a 65 $\mu \mathrm{mEmSafe}$ filter for embryo recovery with integrated Petri dish with a grid (Minitüb COP, Germany). Flushing continued until the embryo was recovered in the filter and was seen by naked eye. Gross evaluation of embryonic shape was carried out as well as microscopic evaluation of embryo capsule and trophoblast cells.

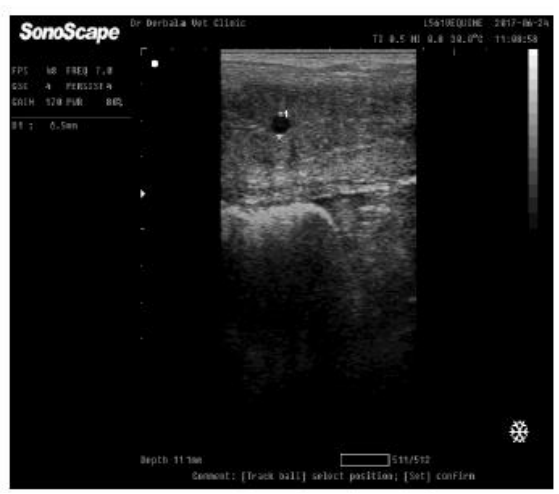

Fig (1-B). Detection of an embryo by ultrasound on day 11 post ovulation

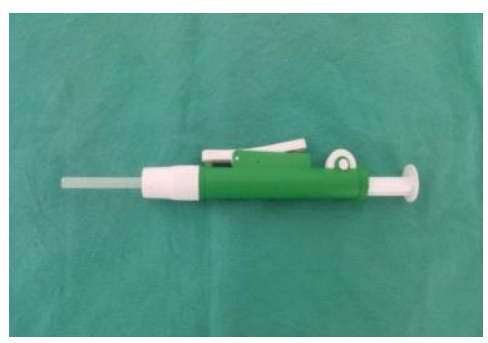

Fig (2-A): A manual propipetter used for loading of embryo into a catheter and release of the embryo into mare's uterus

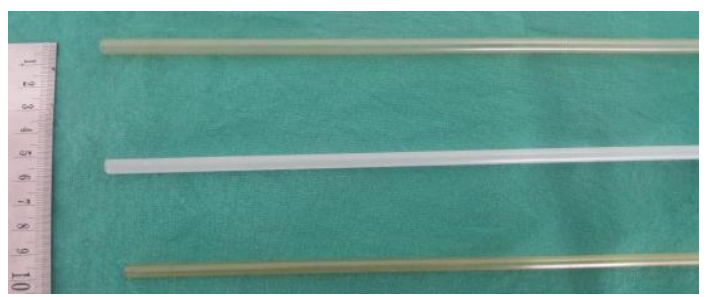

Fig (2-B): Different sizes catheters used for large equine embryo; 1-external sheath of double guarded uterine swab, 2- AI and ET catheter, 3-catheter used for $A I$ 


\subsection{Pregnancy Diagnosis in Recipient Mares}

Pregnancy diagnosis in recipient mares was carried out using transrectal ultrasound scanning. Examinations were done 15, 30, 45, 60 and 90 days post ovulation.

\section{Results}

No flushing was carried to donor mares unless embryonic sac was recorded by ultrasound.

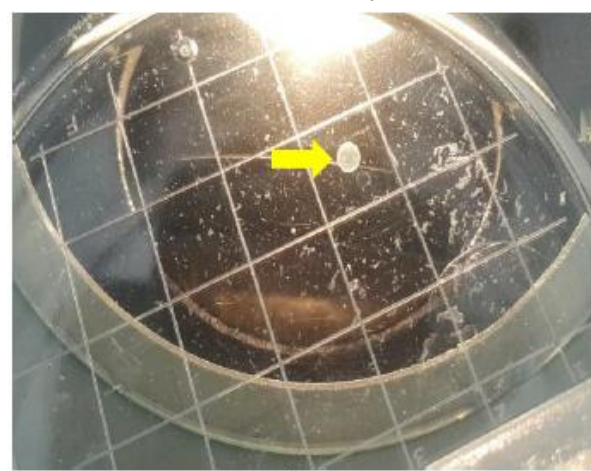

(A)
Control mares (6 mares) were scanned as well for detection of embryonic sacs and monitoring pregnancy. All recovered embryos were large expanded blastocysts with diameters ranged from $4-6.5 \mathrm{~mm}$ (Fig. 3-a, b).The recovery rate was $94.4 \%(34 / 36)$ as there were 2 recovered intact capsules enclosing collapsed trophoblasts of a day 10 and 11 embryos damaged during flushing (Fig. 4-a, b).

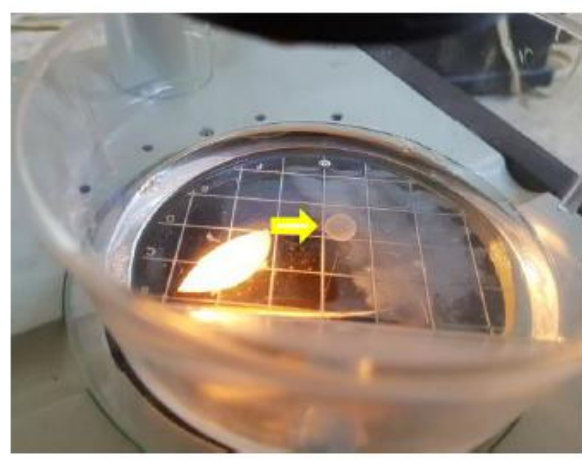

(B)

Fig3. Large expanded blastocyst seen by naked eye on day 10 (A) and on day 11 (B)

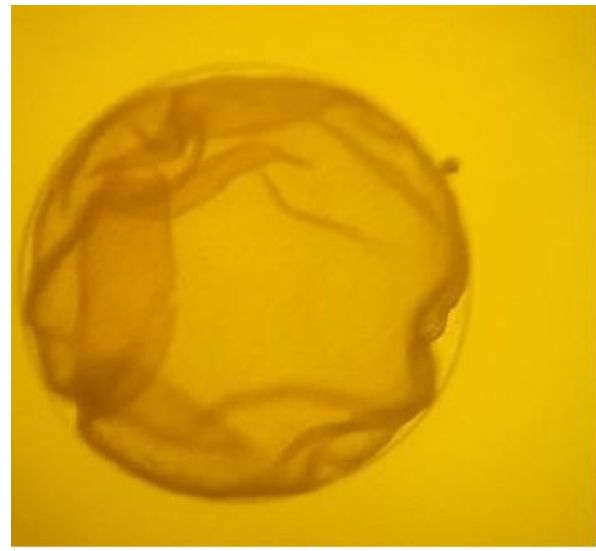

(A)

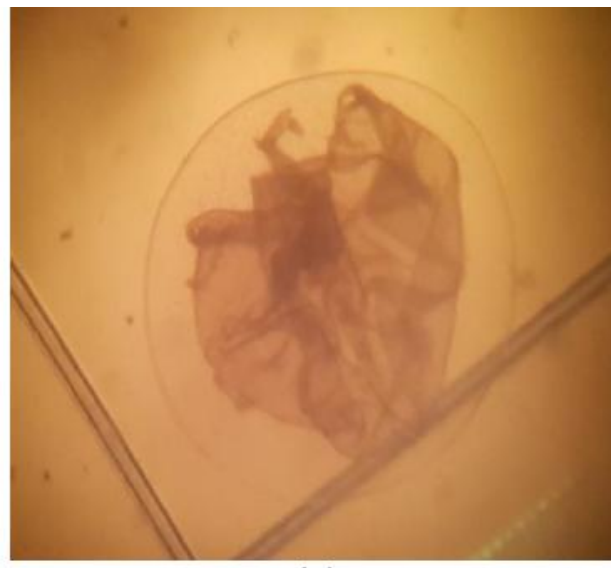

(B)

Fig4. Collapse of the trophoblast within an intact capsule of a day $10(A)$ and day 11 (B) embryos

There were 2 embryos recovered from uteri of mares that have been died suddenly at 11 days of pregnancy, only one of them succeeded posttransfer (Table 1). The success rate post transfer was $73.5 \%$ for embryos transferred at 10 and 11

Table1. Recovery rate, pregnancy rate after transfer and embryonic deaths after success of transfer and formation of embryo proper

\begin{tabular}{|c|c|c|c|c|}
\hline Day of flush & $\begin{array}{c}\text { Number of donor } \\
\text { mares }\end{array}$ & Recovery rate & Pregnancy rate & Embryonic deaths \\
\hline 10 & 16 & $15 / 16$ & $12 / 15$ & 2 \\
\hline 11 & 18 & $17 / 18$ & $12 / 17$ & 1 \\
\hline 11 & 2 (after death) & $2 / 2$ & $1 / 2$ & - \\
\hline & & $94.4 \%(34 / 36)$ & $73.5 \%(25 / 34)$ & $12 \%(3 / 25)$ \\
\hline
\end{tabular}

The recovery rate was similar for 10 and 11 day old embryos. The effect of day of ovulation in recipient mare on pregnancy rate post transfer days post-ovulation. There were $12 \%(3 / 25)$ embryonic deaths observed at 20, 35 and 45 days of pregnancy; so that the conception rate was ultimately $64.7 \%$. 


\begin{tabular}{|c|c|c|}
\hline \multicolumn{2}{|c|}{} \\
\hline Days post ovulation & Pregnancy rate $(\%)$ & NO. \\
\hline 4 & 92.3 & $12 / 13$ \\
\hline 5 & 71.4 & $3 / 14$ \\
\hline 6 & 42.8 & $3 / 7$ \\
\hline
\end{tabular}

\section{DISCUSSION}

In the vast majority of equine embryo transfer programs, the embryo recovery usually carried out on days 6,7 and 8 post ovulation and transferred into recipient mares that ovulated 1 day before to 3 days after the donor mare. The current study differed substantially in that flushing of donor mares was done when embryos were detected by ultrasound scanning (on day 10-11 post ovulation) followed by transfer into a recipient mare depending on using a large pore catheters to fit the embryo size. The current embryo transfer programs do not depend on ultrasographic detection of embryos in the donor mare, so that several flushes should be carried out to get the embryo, which means loss of large volumes of flushing media and stress on mare's uterus through applying several washes, particularly in mares with a history of reduced embryo recovery due to subfertility. Furthermore, in old mares there is a delay in descent of the embryo into the uterus and thus the determination of day of flush is not easy, but once the embryo is detected by ultrasound, the flushing could be performed. So the operator could save flushing media and numbers of flushing until he could realize the presence of a formed embryonic sac.

David Hartman (2011) mentioned that veterinarians tend to flush on day 8 or 9 to visualize embryo in the filter to stop flushing once they detect embryo, but in this practice twins may be lost. Comparatively, in the current study twins could be detected with ultrasonography and flushing continues until obtaining the twins. The present study, therefore, is more practical in recovery of twins.

The studies carried out concerning transfer of old age embryos are few (Allen et al., 1976; Steffenhagen et al., 1972; Iuliano, 1983; Iuliano et al., 1985) Nevertheless, Vogelsang et al. (1985) reported no pregnancy post transfer of day 9 and 10 embryos, whereas Fleury et al (1989) claimed that they obtained 69\% pregnancy rate for embryos collected 9 days post ovulation. These contradictory results might be due to recipient factors or method of transfer. In the current study, the success rate after transfer was $73.5 \%$ although the embryo size was larger (10-11 days) than in previous studies. Based on the present findings, there was a high degree of success in embryo recovery (94.4\%) and pregnancy rate reached $73.5 \%$ compared to the results obtained by Jacob et al. (2012), who got recovery rate $(63 \%)$ and pregnancy rate $(70 \%)$.

Wilsher et al (2010) mentioned a success in transfer in 10 days embryo and the problematic aspect of the method of transfer as well as failure of recovery of embryo $>5 \mathrm{~mm}$ after detection with ultrasonography. The current study succeeded recovery of embryos 4-6.5 mm in diameters, furthermore using different sizes catheters according to the embryo size and using special kind of manual controllable pipettes to manage embryo loading, amount of holding media as well as slow release of the embryo during transfer to avoid embryo burst.

Based on the current findings, embryo transfer of large equine embryos (over 10 days) could be done in mares pregnant 9-11 days who died suddenly or were subjected to euthanasia for urgent reasons.

In conclusion, the results of previous studies concerning transfer of large equine embryo aged between 9 to 10 days were contradictory. The current study emphasized the possibility of transfer of large embryos that could be detected by ultrasound scanning. This method can be used for mares with a history of low embryo recovery to avoid loss of flushing media and decrease the number of flushes. Moreover, the current method can be applied for mares died at 10-11 days of pregnancy or have been subjected to euthanasia. In addition, the technique could be used for mares when the operator missed the day of flushing for any reason or have to delay the time of flushing 1-3 days for availability of a recipient.

\section{ACKNOWLEDGEMENTS}

Thanks are due for all the team of assistants, labors and owners of the farms that helped executing this work.

\section{REFERENCES}

[1] Allen W.R., Stewart F., Trounson A.O., 1976. Variability of horse embryos after storage and long distance transport in the rabbit. J. ReprodFertil. 47, 387-390.

[2] Ball B.A., Little T.V., Weber J.A., Wood G.L., 1989. Survival of Day-4 embryos from young, normal and aged subfertile mares after 
transfer to normal recipient mares.J ReprodFertil.85, 187-209.

[3] Camillo F., Vannozzi I., Rota A., 2002. Age at puberty, cyclicity, clinical response to PGF2 alpha, hCG and GnRH and embryo recovery rate in yearling mares. Theriogenology. 58, 627-630.

[4] Hartman,D.L., 2011. Embryo transfer, Equine reproduction, second edition, Blackwell's publishing, USA, Chapter 303; pp 2871-2879.

[5] Fleury J.J., Neto J.B.F.C., Alvarenga M.A. 1989., Results from an embryo transfer programme with Mangalara mares in Brazil. Equine Vet.J. (Suppl8), 73-4.

[6] Iuliano M.F. (1983). The effect of age of equine embryo and method of transfer on pregnancy rate, Thesis, Fort Collins, Clorado State University.

[7] Iuliano M.F., Squires E.L., Cook V.M., 1985. Effect of age of equine embryos and method of transfer on pregnancy rate. J Anim. Sci. 60, 258-263.

[8] Jacob J.C.F., Haag K.T., Santos G.O., Oliveira J.P., Gastal M.O., Gastal E.L., 2012. Effect of embryo age and recipient asynchrony on pregnancy rates in a commercial equine embryo transfer program.Theriogenology.77, 1159-1166.
[9] Mackinnon O. Angus, Squires L. Edward, 2007. Embryo transfer and related technologies, current therapy in equine reproduction. Ch. 51; pp 319-334.

[10] Squires E.L., Carnevale E.M., McCue P.M., Bruemmer J.E. 2003 Embryo technologies in the horse. Theriogenol. 59, 151-170.

[11] Squires, E.L., Carnevale, E.M., McCue, P.M. and Bruemmer, J.E. 2003. Embryo technologies in the horse. Theriogenology 59, 151-170.

[12] Steffenhagen W.P., Pineda M.H., Ginther O.J., 1972. Retention of unfertilized ova in uterine tubes of mares. Am. J. Vet. Res. 33, 2391-2398.

[13] Stout T. A. E., 2006. Equine embryo transfer: Review of developing potential. Equine vet. J. 38, 467- 478 .

[14] Vogelsang SG, Bondioli KR, Massey JM., 1985. Commercial application of equine embryo transfer. Equine Vet. J. (Suppl 3), 89-91.

[15] Wilsher S., Clutton-Brock Amber, Allen W.R., 2010.Successful transfer of day 10 horse embryos: influence of donor-recipient asynchrony on embryo development. Reprod. Res. 139, 575-585.

Citation: M.K. Derbala, M.S.S. Abdou, Transfer of Large Equine Embryos in Arabian Mares. ARC Journal of Animal and Veterinary Sciences. 2017; 3(4):19-24. doi: dx.doi.org/ 10.20431/2455-2518.0304003.

Copyright: (C) 2017 Authors. This is an open-access article distributed under the terms of the Creative Commons Attribution License, which permits unrestricted use, distribution, and reproduction in any medium, provided the original author and source are credited. 